\title{
Colorectal Cancer and Polyps in Diverticulosis Patients: A 10-Year Retrospective Study in 13680 Patients
}

\author{
Fadi Abu Baker $\mathbb{D}^{1},{ }^{1}$ Jesus Alonso Z'cruz De La Garza, ${ }^{2}$ Amir Mari $\mathbb{D}^{3}{ }^{3}$ Abdel-Rauf Zeina, ${ }^{4}$ \\ Amani Bishara, ${ }^{1}$ Oren Gal, ${ }^{1}$ and Yael Kopelman ${ }^{1}$ \\ ${ }^{1}$ Department of Gastroenterology and Hepatology, Hillel Yaffe Medical Center, Affiliated to the Technion Faculty of Medicine, \\ Hadera, Haifa, Israel \\ ${ }^{2}$ Department of Surgery, Hillel Yaffe Medical Center, Affiliated to the Technion Faculty of Medicine, Hadera, Haifa, Israel \\ ${ }^{3}$ Department of Gastroenterology, Nazareth EMMS Hospital, Affiliated to the Faculty of Medicine, Bar Ilan University, Israel \\ ${ }^{4}$ Department of Radiology, Hillel Yaffe Medical Center, Affiliated to the Faculty of Medicine, Technion-Israel Institute of Technology, \\ Haifa, Israel
}

Correspondence should be addressed to Fadi Abu Baker; fa_fd@hotmail.com

Received 31 August 2019; Revised 7 October 2019; Accepted 29 October 2019; Published 1 December 2019

Academic Editor: Gabriela Melen-Mucha

Copyright (C) 2019 Fadi Abu Baker et al. This is an open access article distributed under the Creative Commons Attribution License, which permits unrestricted use, distribution, and reproduction in any medium, provided the original work is properly cited.

\begin{abstract}
Introduction. Shared by certain epidemiological and etiological characteristics, diverticulosis and colorectal cancer (CRC) as well as colonic polyps has long been linked. This association was studied in several heterogeneous studies but has reported inconsistent results. Clarifying the association is clinically relevant for endoscopist awareness and potential modification of screening and surveillance intervals for diverticulosis patients. Methods. In this retrospective single-center study, patients diagnosed with diverticulosis on colonoscopy over a 10-year period were included. Each diverticulosis patient was matched with 1 control by age, gender, setting (inpatient/outpatient), and procedure's indication. CRC and polyp detection rates were recorded and compared between the groups before and after adjustment for bowel preparation quality and exam completion. CRC location was recorded and compared between groups. Results. A cohort of 13680 patients (6840 patients with diverticulosis and 6840 matched controls) was included. Diverticulosis was located mainly to the sigmoid and left colon (94.4\%). The CRC diagnosis rate was lower in the diverticulosis group ( $2 \%$ vs. $4.5 \%$, odds ratio $=0.472, P<0.001$, and $95 \% \mathrm{CI}=0.382-0.584)$. Moreover, location of CRC was unrelated to diverticulosis location, as more CRCs in the diverticulosis group were located proximal to the splenic flexure as compared to the control group ( $42.5 \%$ vs $29.5 \%$, respectively; $P=0.007)$. Diverticulosis, however, was associated with an increased polyp detection rate compared to controls $(30.5 \%$ vs. $25.5 \%$; odds ratio $=1.2, P<0.001$, and $95 \%$ $\mathrm{CI}=1.11$-1.299). Conclusion. We demonstrated that diverticulosis was not associated with an increased risk for CRC. A possible increased polyp detection rate, however, warrants further evaluation in large prospective studies.
\end{abstract}

\section{Background}

Diverticulosis is considered as one of the most common and burdensome GI disorders [1,2]. The underlying pathological mechanisms resulting in diverticular formation of a colonic wall is still largely unknown. Diverticula develop at welldefined points of weakness in the circular muscle of colon and are likely to be the result of complex interactions between environmental and heritable factors including diet, increased age, and decreased colonic motility, among others $[3,4]$. Typically, diverticulosis is identified incidentally at colonoscopy or imaging studies performed for various indications, and the majority of patients with diverticulosis remain asymptomatic throughout their lifetime [5].

Several observations hold that certain epidemiological and etiological characteristics are shared between colonic diverticulosis and colorectal cancer, suggesting a possible association between these two conditions. The prevalence of these conditions is markedly on the rise in the last decades, and they both are detected frequently in aged people as well as western population and industrialized countries [6-8]. Western diet, namely, low dietary fiber and high total fat, 
has been largely regarded to play a major role in the pathogenesis and was epidemiologically associated with an increased risk of both conditions [9-13].

The abovementioned connection is of great clinical relevance, as several reports demonstrated that patients with diverticular disease have a higher risk of harboring colonic cancer [14-16]. Above and beyond, one meta-analysis demonstrated that diverticular disease was associated as well with increased detection of colorectal adenomas [17].

However, data are still controversial and inconclusive as other recent studies failed to confirm this association [18-20]. Moreover, the vast majority of the studies inspecting a possible link between both conditions were limited by small patient numbers and did not account for multiple confounders that are known to affect CRC and polyp detection such as patients' demographics, procedure's indication, setting, quality of bowel preparation, and exam completion.

Taken together, unraveling the dilemma and clarifying the association between both conditions appear to be clinically relevant, as modifying screening or surveillance intervals for CRC and polyp follow-up may be warranted accordingly in patients with diverticular disease.

The present study is aimed at comparing the CRC diagnosis rate and location as well as polyp detection rate between patients with diverticular disease and a matched group without diverticulosis with adjustment for abovementioned confounders.

\section{Methods and Settings}

We conducted a retrospective, large cohort study, which examined consecutive patients who underwent colonoscopies over a 10-year period within the gastroenterology department at the Hillel Yaffe Medical Center, a universityaffiliated hospital in Israel. All patients' data were collected from our department's electronic record system. We searched endoscopy reports to identify all patients with a diagnosis of diverticulosis to create a study group. For a control group, each patient from the study group was matched with 1 control patient by age, gender, setting (inpatient/outpatient), and procedure's indication. Patients were excluded if they were less than 18 years, had prior diagnosis of colon cancer, or if full data set is missing. Endoscopy findings including cancer diagnosis and location as well as polyp detection were recorded in both groups. Diverticulosis location was documented as well in the diverticulosis group. Whenever an endoscopic diagnosis of colorectal cancer was encountered, histology reports were reviewed to confirm diagnosis.

We compared the rate of CRC and polyp diagnosis between both groups and use multivariable analysis to adjust for adequacy of bowel preparation (adequate/inadequate) and depth of examination (cecal intubation confirmed or not), in order to identify independent association of diverticulosis with CRC and polyp detection. CRC location was documented according to endoscopy reports, and we compared its location between both groups. Diverticulosis and CRC location was classified as proximal (proximal to splenic flexure) or distal (splenic flexure or distal). The local institutional
Helsinki ethics board approved the study and granted exemption from informed consent in this retrospective study as patients were receiving standard care without relation to the study.

\section{Statistical Analysis}

This statistical analysis is dealing with cohort of "big data" (40128 patients), of them 6840 patients with diverticulosis (study group). We used the Propensity Score Matching in $\mathrm{R}$ program version 3.3 to divide the total cohort to $1: 1$ ratio (study and control group). Descriptive statistics in terms of mean, SD, and percentiles were preformed to the whole parameters in the study. Differences between the two groups (diverticulosis diagnosed vs. matched group) in the quantitative parameters were demonstrated by $t$-test. For the categorical parameters, we used fisher exact tests. Multivariate logistic regression model was used to determine the effect of the independent parameters associated with CRC. SPSS version 25 was also used for statistical analysis. $P<0.05$ was considered as significant.

\section{Results}

We included a large cohort of 40128 patients who underwent colonoscopy at our hospital. We searched endoscopy reports and identified 6840 patients (17\%) with diverticulosis diagnosed during the study period. A matched group of 6840 control patients (at $1: 1$ ratio) was included for final analysis. Baseline characteristics of both groups were similar and are provided in Table 1. The overall mean age was $68.3 \pm 11.0$ years (range: $18-101$ ), with a slight male predominance $(52.1 \%)$. The vast majority of the procedures $(78.4 \%)$ were performed in the outpatient setting. Procedures' indications did not differ significantly between groups. The most common indications for colonoscopy were abdominal pain and diarrhea $(21.1 \%)$, rectal bleeding (14.2\%), and anemia (13.2\%).

The CRC diagnosis rate was lower in the diverticulosis group ( $2 \%$ vs. $4.5 \%$; $P<0.01)$ while the polyp detection rate was surprisingly higher $(30.5 \%$ vs. $25.5 \% ; P<0.01)$ as compared to the matched group (Table 2). Cecal intubation rate (92.8\% vs. $84.1 \% ; P<0.01$ ), adequate bowel preparation rate $(90.7 \%$ vs. $84.1 \% ; P<0.01)$, and terminal ileum intubation rate $(2.2 \%$ vs. $1.6 \%$; $\mathrm{OR}=1.4$ and $95 \% \mathrm{CI}=1.096-1.805$; $P=0.008)$ were significantly higher in the diverticulosis group (Table 2). Multivariate analysis (Table 3) to account for these variables revealed similar trends as diverticulosis patients were associated with less CRC diagnosis (odds ratio $=0.472, P<0.001$, and $95 \% \mathrm{CI}=0.382-0.584$ ) but increased polyp detection rate (odds ratio $=1.2, P<0.001$, and $95 \% \mathrm{CI}=1.11-1.299)$.

Diverticulosis and CRC locations are demonstrated in Table 4. Diverticulosis was located mainly to the distal colon (94.4\%). Location of CRC was unrelated to diverticulosis location, as more CRCs in the diverticulosis group were located proximally compared to the control group $(42.5 \%$ vs. $29.5 \%$, respectively; $P=0.07)$. 
TABLE 1: Baseline characteristics of diverticulosis and matched groups.

\begin{tabular}{lccc}
\hline Characteristics & Matched group $(N=6840)$ & Diverticulosis group $(N=6840)$ & $P$ value \\
\hline Age at test (years) & $68.3 \pm 11.0$ & $68.3 \pm 11.0$ & $3=0.90$ \\
Gender (male) & $3570(52.2 \%)$ & $3566(52.1 \%)$ & \\
Setting (outpatient) & $5365(78.5 \%)$ & & \\
Procedures' indication & & $641(9.4 \%)$ & 0.93 \\
Personal history of polyps & $657(9.6 \%)$ & $1454(21.3 \%)$ & $P=0.69$ \\
Abdominal pain/diarrhea & $1432(20.9 \%)$ & $44(0.6 \%)$ & $P=0.64$ \\
IBD follow-up & $41(0.6 \%)$ & $187(2.7 \%)$ & $P=0.66$ \\
Past colonic surgery & $189(2.8 \%)$ & $922(13.5 \%)$ & $P=0.83$ \\
Anemia & $876(12.8 \%)$ & $921(7.6 \%)$ & $P=0.92$ \\
Positive FOBT & $497(7.3 \%)$ & $994(14.5 \%)$ & $P=0.26$ \\
Rectal bleed & $947(13.8 \%)$ & $416(6.1 \%)$ & $P=0.45$ \\
Family history of CRC & $400(5.8 \%)$ & $254(3.7 \%)$ & $P=0.26$ \\
Screening & $248(3.6 \%)$ & $777(11.4 \%)$ & $P=0.59$ \\
Constipation & $747(10.9 \%)$ & $382(5.6 \%)$ & $P=0.82$ \\
Imaging findings & $394(5.8 \%)$ & $237(3.5 \%)$ & $P=0.66$ \\
Weight loss & $185(2.7 \%)$ & $P=0.12$ &
\end{tabular}

TABLE 2: Endoscopic findings in both groups.

\begin{tabular}{lccc}
\hline Endoscopic findings & Matched group $(N=6840)$ & Diverticulosis group $(N=6840)$ & $P$ value \\
\hline Polyp detection rate & $1747(25.5 \%)$ & $2083(30.5 \%)$ & $140(2.0 \%)$ \\
CRC diagnosis rate & $306(4.5 \%)$ & $6349(92.8 \%)$ & $P<0.001$ \\
Complete procedure & $5750(84.1 \%)$ & $6205(90.7 \%)$ & $P<0.001$ \\
Adequate bowel preparation & $5748(84.1 \%)$ & $151(2.2 \%)$ & $P<0.001$ \\
Terminal ileum intubation & $108(1.6 \%)$ & $P=0.008$ \\
\hline
\end{tabular}

\section{Discussion}

The current study was designed to clarify several aspects of diverticulosis and its possible association with CRC and polyp diagnosis. This association has long been studied in observational, cross-sectional, and case-control studies and has reported inconsistent results. Moreover, small patient numbers and heterogeneous study design contributed to conflicting conclusions. In the current study, we included a large cohort over a 10-year period and performed group matching followed with multivariate analysis in order to account for as many confounders as possible that may have influenced results of preceding studies.

We found that diverticulosis patients were not associated with an increased rate of CRC diagnosis compared to a matched group ( $2 \%$ vs. $4.5 \%$; OR $=0.472$, $95 \% \mathrm{CI}=0.382$ $0.584, P<0.001)$. Our findings confirm the findings from other recent studies that diverticulosis is not associated with increased CRC diagnosis. A nationwide case-control study found that diverticular disease does not increase the risk of colon cancer in the long term, and a history of diverticular disease does not affect colon cancer mortal- ity [18]. Our findings are also in concordance with a study by Meurs-Szojda et al. on more than 4200 colonoscopies which demonstrated a negative correlation between colon cancer and diverticulosis [21].

Moreover, we provided detailed location of diverticulosis and colon cancer in our cohort. Similar to different reports in western population $[6,7,9]$, diverticulosis was located predominantly to the distal colon as more than $94 \%$ of diverticula were located to sigmoid and descending colon. In this regard, not only we demonstrated that CRC was located into sigmoid and descending colon in less than $35 \%$ but also we showed that more CRCs in the diverticulosis group were located proximally compared to the control group (Table 3) (42.5\% vs. $29.5 \%$, respectively; $P=0.07$ ). Consistent findings were reported by Cooper et al. who showed that diverticulosis associated interval cancers were somewhat more likely to be in the proximal colon and less likely to be in the distal colon [15]. Taken together, these findings reinforce the conclusion that CRC and diverticulosis are unrelated.

One worth mentioning finding in this study, however, is the increased polyp detection rate in diverticulosis patients (30.5\% vs. $25.5 \%$; $\mathrm{OR}=1.2,95 \% \mathrm{CI}=1.11-1.299, P<0.01$ ). 
TABLE 3: Risk factors for colorectal cancer diagnosis, a multivariate analysis.

\begin{tabular}{lccc}
\hline Characteristics & $P$ value & Odds ratio & Upper \\
\hline Age at test (years) & $<0.01$ & 1.042 & 1.031 \\
Gender (male) & 0.357 & 1.099 & 0.899 \\
Group (diverticulosis) & $<0.01$ & 0.472 & 0.382 \\
Incomplete exam & $<0.01$ & 0.294 & 0.232 \\
Procedures' indication & & & 0.584 \\
Personal history of polyps & 0.102 & 1.344 \\
Abdominal pain/diarrhea & 0.954 & 0.990 & 0.373 \\
IBD follow-up & 0.300 & 1.138 & 0.703 \\
Past colonic surgery & 0.299 & 0.612 & 0.892 \\
Anemia & $<0.01$ & 0.242 & 2.362 \\
Positive FOBT & $<0.01$ & 2.912 & 2.168 \\
Rectal bleed & $<0.01$ & 2.830 & 1.393 \\
Family history of CRC & 0.506 & 3.504 & 1.452 \\
Screening & 0.350 & 1.066 & 1.546 \\
Constipation & 0.077 & 0.572 & 3.911 \\
Imaging findings & $<0.01$ & 0.672 & 4.210 \\
Weight loss & 0.269 & 8.594 & 4.803 \\
\hline
\end{tabular}

TABLE 4: Diverticulosis and colorectal cancer location in both groups.

\begin{tabular}{|c|c|c|c|c|}
\hline \multirow{2}{*}{ Location } & & \multicolumn{2}{|c|}{ Diverticulosis group } & \multirow{2}{*}{ Matched group } \\
\hline & & $\mathrm{CRC}$ & Diverticulosis* & \\
\hline \multirow{4}{*}{ Distal colon } & Rectum & $0 \%$ & $20 \%$ & $23.90 \%$ \\
\hline & Rectosigmoid & $2.6 \%$ & $7.60 \%$ & $14 \%$ \\
\hline & Sigmoid & $84.1 \% *$ & $16.70 \%$ & $17.30 \%$ \\
\hline & Descending colon & $23 \%^{* *}$ & $11 \%$ & $14 \%$ \\
\hline \multirow{4}{*}{ Proximal colon } & Transverse colon & $2.6 \%$ & $4.90 \%$ & $3.70 \%$ \\
\hline & Ascending colon & $5 \%{ }^{* * *}$ & $27.80 \%$ & $17.90 \%$ \\
\hline & Cecum & $3 \%^{* * * *}$ & $9.80 \%$ & $7.30 \%$ \\
\hline & Undetermined & $0 \%$ & $2.20 \%$ & $2 \%$ \\
\hline
\end{tabular}

${ }^{*}$ Sigmoid (alone: $65 \%$, sigmoid+descending: $12.5 \%$, sigmoid+transverse: $2.6 \%$, sigmoid+ ascending/cecum: $4 \%$ ). ${ }^{* *}$ Descending colon (alone: $10.5 \%$, sigmoid +descending: 12.5\%). ${ }^{* * *}$ Ascending colon (alone: $2 \%$, sigmoid+ascending: $3 \%$ ). ${ }^{* * * *}$ Cecum (alone 1\%, sigmoid+cecum: $2 \%$ ).

This observation is supported by several other studies reporting that patients with diverticulosis have a higher risk of colorectal polyps as compared to those without [22, 23]. One meta-analysis found a significant 1.67-fold increased odds of developing adenomas in patients with diverticulosis [17]. Unfortunately, we were unable to determine the location and histologic type of these polyps in the current study. However, given the lower CRC diagnosis rate in the diverticulosis patients, this may point out that the majority of the detected polyps were of low dysplastic progression potentials (diminutive/hyperplastic polyps), thus explaining the low CRC diagnosis albeit the high PDR. Nevertheless, this observation needs further validation by a large prospective cohort study.

Furthermore, we demonstrated that diverticulosis has no significant effect on the outcome of colonoscopy. Linked with suboptimal bowel preparation, it is thought that diverticulo- sis may cause technical difficulty to perform a complete colonoscopy as a result of a spastic colon and luminal narrowing [24-26]. However, we demonstrated the contrary as quality indicators such as the cecal intubation rate $(92.8 \%$ vs. $84.1 \% ; \mathrm{OR}=2.45$ and $95 \% \mathrm{CI}=2.187-2.738 ; P<0.01)$ and the adequate bowel preparation rate $(90.7 \%$ vs. $84.1 \%$; $\mathrm{OR}=1.853$ and $95 \% \mathrm{CI}=1.670-2.057 ; P<0.01)$ as well as the terminal ileum intubation rate $(2.2 \%$ vs. $1.6 \%$; $\mathrm{OR}=1.4$ and $95 \% \mathrm{CI}=1.096-1.805 ; P=0.008)$ which were even better in diverticulosis patients compared to those without. Similar findings were reported by Gohil et al. who found that diverticulosis did not adversely affect the cecal intubation rate, withdrawal times, or sedation requirements [27].

One of the strengths of the current study includes the large number of participants involved as well as the inclusion of multiple factors such as procedures' settings and 
indications reflecting real daily practice. Our study has limits inherent in its retrospective nature. Besides, other possible factors that may have affected endoscopy findings such as withdrawal time and variable endoscopist experience could not be obtained and were not included.

In conclusion, diverticulosis apparently is not linked with an increased risk of CRC but is possibly associated with an increased polyp detection rate. Prospective studies to clarify these findings are warranted.

\section{Data Availability}

The datasets used and/or analyzed during the current study are available from the corresponding author on reasonable request.

\section{Conflicts of Interest}

The authors certify that they have no affiliations with or involvement in any organization or entity with any financial or nonfinancial interest in the subject matter or materials discussed in this manuscript. The authors declared no potential conflicts of interest with respect to the research, authorship, and/or publication of this article.

\section{References}

[1] N. J. Shaheen, R. A. Hansen, D. R. Morgan et al., "The burden of gastrointestinal and liver diseases, 2006," The American Journal of Gastroenterology, vol. 101, no. 9, pp. 2128-2138, 2006.

[2] J. E. Everhart and C. E. Ruhl, "Burden of digestive diseases in the United States part II: lower gastrointestinal diseases," Gastroenterology, vol. 136, no. 3, pp. 741-754, 2009.

[3] T. P. Bhucket and N. H. Stollman, "Diverticular disease of the colon," in Sleisenger and Fordtran's Gastrointestinal and Liver Disease: Pathophysiology, Diagnosis, Management, M. Feldman, L. S. Friedman, and L. J. Brandt, Eds., vol. 2, pp. 1-15, Elsevier, Philadelphia, 10th edition, 2014.

[4] A. Tursi, A. Papa, and S. Danese, "Review article: the pathophysiology and medical management of diverticulosis and diverticular disease of the colon," Alimentary Pharmacology \& Therapeutics, vol. 42, no. 6, pp. 664-684, 2015.

[5] J. Hemming and M. Floch, "Features and management of colonic diverticular disease," Current Gastroenterology Reports, vol. 12, no. 5, pp. 399-407, 2010.

[6] J. Y. Kang, J. Hoare, A. Tinto et al., "Diverticular disease of the colon-on the rise: a study of hospital admissions in England between 1989/1990 and 1999/2000," Alimentary Pharmacology \& Therapeutics, vol. 17, no. 9, pp. 1189-1195, 2003.

[7] D. A. Etzioni, T. M. Mack, R. W. Beart Jr., and A. M. Kaiser, "Diverticulitis in the United States: 1998-2005: changing patterns of disease and treatment," Annals of Surgery, vol. 249, no. 2, pp. 210-217, 2009.

[8] A. F. Peery, T. O. Keku, C. F. Martin et al., "Distribution and characteristics of colonic diverticula in a United States screening population," Clinical Gastroenterology and Hepatology, vol. 14, no. 7, pp. 980-985.e1, 2016.

[9] N. Painter and D. Burkitt, "Diverticular disease of the colon: a deficiency disease of Western civilization," British Medical Journal, vol. 2, no. 5759, pp. 450-454, 1971.
[10] J. S. Gear, A. Ware, P. Fursdon et al., "Symptomless diverticular disease and intake of dietary fibre," The Lancet, vol. 1, no. 8115, pp. 511-514, 1979.

[11] W. H. Aldoori, E. L. Giovannucci, E. B. Rimm, A. L. Wing, D. V. Trichopoulos, and W. C. Willett, "A prospective study of diet and the risk of symptomatic diverticular disease in men," The American Journal of Clinical Nutrition, vol. 60, no. 5, pp. 757-764, 1994.

[12] F. L. Crowe, P. N. Appleby, N. E. Allen, and T. J. Key, "Diet and risk of diverticular disease in Oxford cohort of European Prospective Investigation into Cancer and Nutrition (EPIC): prospective study of British vegetarians and non-vegetarians," $B M J$, vol. 343 , no. 4 , article d4131, 2011.

[13] D. Aune, D. S. Chan, R. Lau et al., "Dietary fibre, whole grains, and risk of colorectal cancer: systematic review and doseresponse meta-analysis of prospective studies," BMJ, vol. 343, no. 1 , article d6617, 2011.

[14] T. Stefánsson, A. Ekbom, P. Sparèn, and L. Påhlman, "Increased risk of left sided colon cancer in patients with diverticular disease," Gut, vol. 34, no. 4, pp. 499-502, 1993.

[15] G. S. Cooper, F. Xu, M. D. Schluchter, S. M. Koroukian, and J. S. Barnholtz Sloan, "Diverticulosis and the risk of interval colorectal cancer," Digestive Diseases and Sciences, vol. 59, no. 11, pp. 2765-2772, 2014.

[16] L. Q. Mortensen, J. Burcharth, K. Andresen, H. C. Pommergaard, and J. Rosenberg, "An 18-year nationwide cohort study on the association between diverticulitis and colon cancer," Annals of Surgery, vol. 265, no. 5, pp. 954-959, 2017.

[17] V. Jaruvongvanich, A. Sanguankeo, K. Wijarnpreecha, and S. Upala, "Risk of colorectal adenomas, advanced adenomas and cancer in patients with colonic diverticular disease: systematic review and meta-analysis," Digestive Endoscopy, vol. 29, no. 1, pp. 73-82, 2017.

[18] J. Granlund, T. Svensson, F. Granath et al., "Diverticular disease and the risk of colon cancer - a population-based casecontrol study," Alimentary Pharmacology \& Therapeutics, vol. 34, no. 6, pp. 675-681, 2011.

[19] W. Y. Huang, C. C. Lin, Y. M. Jen et al., "Association between colonic diverticular disease and colorectal cancer: a nationwide population-based study," Clinical Gastroenterology and Hepatology, vol. 12, no. 8, pp. 1288-1294, 2014.

[20] S. Morini, A. Zullo, C. Hassan, S. Tomao, and S. M. Campo, "Diverticulosis and colorectal cancer: between lights and shadows," Journal of Clinical Gastroenterology, vol. 42, no. 7, pp. 763-770, 2008.

[21] M. M. Meurs-Szojda, "Diverticulosis and diverticulitis form no risk for polyps and colorectal neoplasia in 4,241 colonoscopies," International Journal of Colorectal Disease, vol. 23, no. 10, pp. 979-984, 2008.

[22] A. Muhammad, O. Lamendola, A. Daas, A. Kumar, and G. Vidyarthi, "Association between colonic diverticulosis and prevalence of colorectal polyps," International Journal of Colorectal Disease, vol. 29, no. 8, pp. 947-951, 2014.

[23] S. Morini, C. Hassan, A. Zullo et al., "Diverticular disease as a risk factor for sigmoid colon adenomas," Digestive and Liver Disease, vol. 34, no. 9, pp. 635-639, 2002.

[24] T. N. Witte and R. Enns, "The difficult colonoscopy," Canadian Journal of Gastroenterology, vol. 21, no. 8, pp. 487-490, 2007. 
[25] J. C. Anderson, C. R. Messina, W. Cohn et al., "Factors predictive of difficult colonoscopy," Gastrointestinal Endoscopy, vol. 54, no. 5, pp. 558-562, 2001.

[26] B. P. Saunders, S. Halligan, C. Jobling et al., "Can barium enema indicate when colonoscopy will be difficult?," Clinical Radiology, vol. 50, no. 5, pp. 318-321, 1995.

[27] V. B. Gohil, J. T. Patrie, V. M. Shami et al., "Colonic diverticulosis is associated with an increased adenoma detection rate in patients undergoing first-time screening colonoscopy," Journal of Interventional Gastroenterology, vol. 2, no. 2, pp. 70-75, 2012 Apr-Jun. 


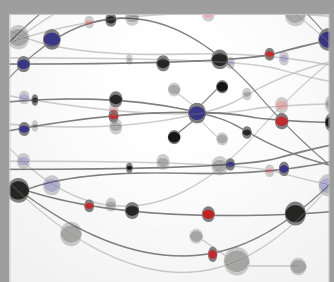

The Scientific World Journal
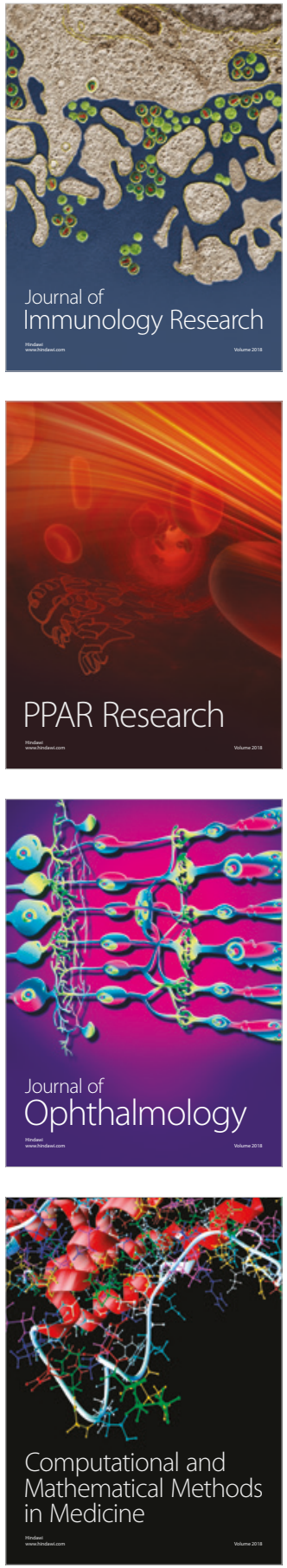

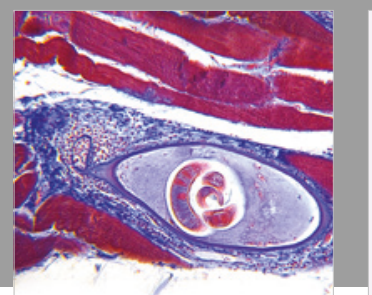

Gastroenterology Research and Practice

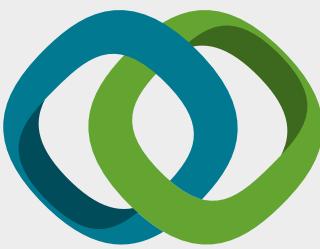

\section{Hindawi}

Submit your manuscripts at

www.hindawi.com
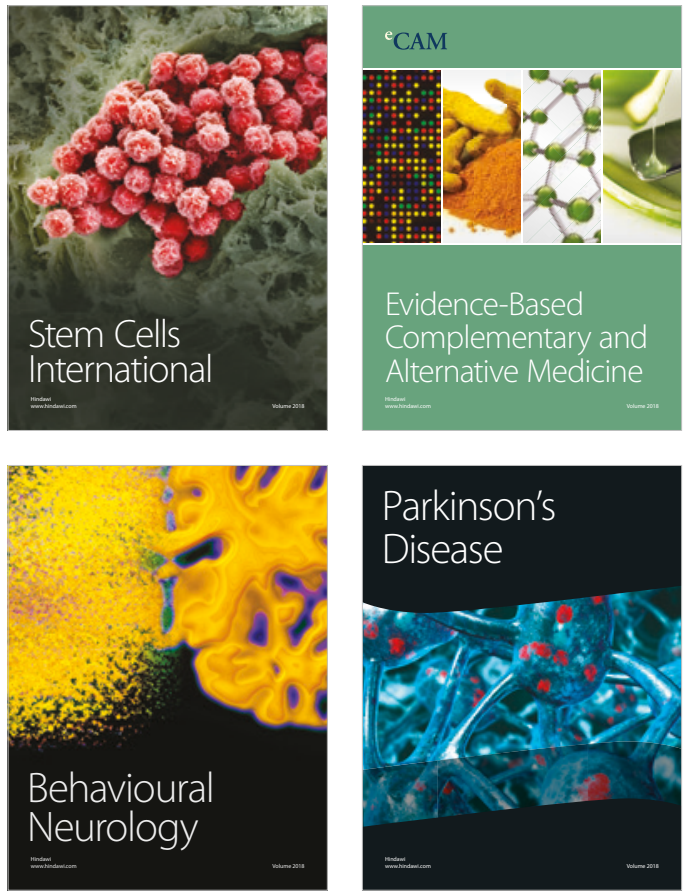

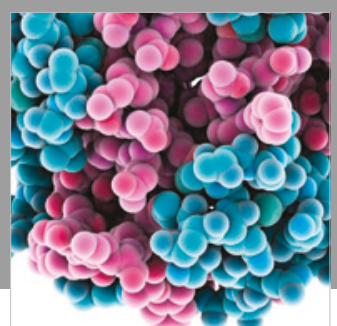

ournal of

Diabetes Research

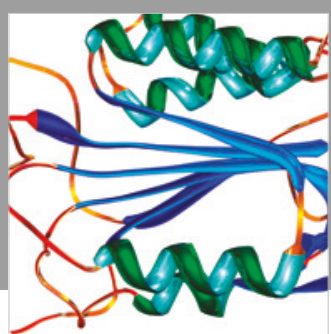

Disease Markers
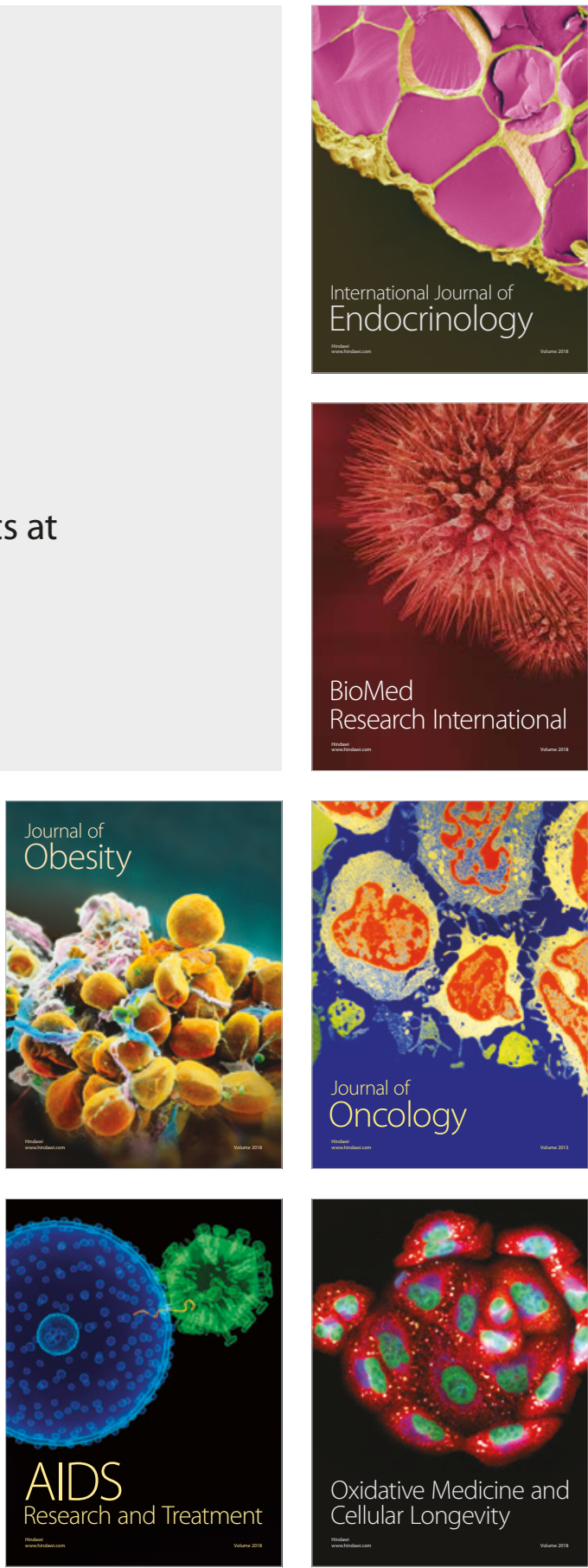